\title{
Lubich-Collocation Method for Solving a System of Nonlinear Integral Equations of Convolution Type
}

\author{
Reza Zolfaghari
}

\begin{abstract}
Lubich convolution quadrature formulas have the fundamental property of not using explicitly the expression of the kernel of the integral equation they are applied to, which is instead replaced by that of its Laplace transform, usually given by a simple analytic function. In this paper, the Lubichcollocation procedure was successfully employed to reduce a system of nonlinear Volterra integral equations with convolution kernels to a system of algebraic equations. An example is considered to illustrate the ability of the proposed method.
\end{abstract}

Index Terms-Nonlinear integral equation, Convolution kernel, Lubich quadrature.

\section{INTRODUCTION}

Many physical, chemical, and biological problems are modeled as Volterra integral equations (VIEs), such as heat conduction problem, concrete problem of mechanics or physics, on the unsteady poiseuille flow in a pipe, diffusion problems, electroelastic, contact problems, etc [1]. We consider the system of nonlinear volterra integral equations of the form

$$
\sum_{j=1}^{M} q_{i j}(x) f_{j}(x)=g_{i}(x)+\sum_{j=1}^{M} \int_{0}^{x} k_{i j}(x-t) \Phi_{i j}\left(t, f_{j}(t)\right) d t,
$$

for $i=1,2, \ldots, M$ where $x \in(0, b)$, the functions $q_{i j}, g_{i}, k_{i j}$ and $\Phi_{i j}$ are known and $f_{i}$ to be determined.

In the last years, several authors have used Lubich convolution quadrature formulas to discretize space-time boundary integral equations representing time dependent problems. Lubich convolution quadrature methods were proposed, implemented and analyzed by Christian Lubich in [1], [2]. From the beginning, a main motivation for considering these methods was that they enjoy excellent stability properties when used for the discretization of integral equations or integro-differential equations of convolution type, in a way often strikingly opposed to standard quadrature formulas using values of the convolution kernel or product integration formulas using moments of the convolution kernel over short intervals [3]. The stability aspect of convolution quadrature methods was emphasized in [4] for Abel-Volterra integral equations, for a large class of nonlinear convolution equations in [5].

The plan of the paper is as follows. In Section II, the Lubich convolution quadrature is summarized. In Section III,

Manuscript received November 9, 2013; revised January 15, 2014.

R. Zolfaghari is with the Department of Computer Science in Salman Farsi University of Kazerun, Iran (e-mail: zolfaghari@kazerunsfu.ac.ir). we use this quadrature method to replace the integral equations (1) by a system of nonlinear algebraic equations. Finally, in Section V, we give some numerical results.

\section{LUBich CONVOLUTION QUADRATURE}

A convolution integral of the form

$$
f(t)=\int_{0}^{t} k(t-\tau) g(\tau) d \tau,
$$

can be approximated by the operational quadrature method developed by Lubich [1], [2].

Assumes that the function $k(t)$ is such that its Laplace transform $K(s)$ is sectorial, i.e. it is analytic in the sector $|\arg (s-c)|<\pi-\phi$, with $\phi<\frac{\pi}{2}, c \in \mathbb{R}$, and

$$
|K(s)| \leq M|s|^{-\mu},
$$

for some real $\mu$ and $M$.

Substituting $k(t)$ by the inverse Laplace transformation of $K(s)$ in the convolution integral (2) and reversing the order of integration leads to

$$
f^{1}(t)=\frac{\lim _{2 \pi i}}{2 \pi i} \int_{c-i R}^{c+i R} K(s) y(t ; s) d s,
$$

where $y(t ; s)=\int_{0}^{t} e^{s(t-\tau)} g(\tau) d \tau$. Setting $y(t)=y(t ; s)$, this function is the solution of the initial value problem

$$
y^{\prime}=s y+g, \quad y(0)=0 .
$$

Therefore, $y(t)$ can be approximated by applying a linear multistep method

$$
y(t) \approx \sum_{j=0}^{l} \alpha_{j} y_{n-j}=h \sum_{j=0}^{l} \beta_{j}\left(s y_{n-j}+g((n-j) h)\right),
$$

with equal time steps $h$ and the starting values $y_{-l}=\ldots=y_{-1}=0$. Taking a representation with formal power series for $y(z)=\sum_{n=0}^{\infty} y_{n} z^{n}$ and $g(z)=\sum_{n=0}^{\infty} g(n h) z^{n}$, the multistep method becomes

$$
\left(\frac{\gamma(z)}{h}-s\right) \sum_{n=0}^{\infty} y_{n} z^{n}=\sum_{n=0}^{\infty} g(n h) z^{n},
$$

where

$$
\gamma(z)=\frac{\alpha_{0}+\alpha_{1} z+\ldots+\alpha_{l} z^{l}}{\beta_{0}+\beta_{1} z+\ldots+\beta_{l} z^{l}} .
$$


The used multistep method should be $A(\alpha)$ stable with $\alpha>\phi$ stable in a neighborhood of infinity, strongly zero-stable and consistent of order $p$. Well-known examples of possible generating polynomials are the backward differentiation formulas of order $p=1,2, \ldots, 6$, given by

$$
\gamma(z)=\sum_{i=1}^{p} \frac{1}{i}(1-z)^{i}
$$

Using Cauchy's integral formula, the resulting approximation of (1) is

$$
\sum_{n=0}^{\infty} f_{n} z^{n}=K\left(\frac{\gamma(z)}{h}\right) \sum_{n=0}^{\infty} g(n h) z^{n}
$$

Representing the function $K(s)$ by a power series

$$
K\left(\frac{\gamma(z)}{h}\right)=\sum_{n=0}^{\infty} \kappa_{n}(h) z^{n}
$$

with the coefficients

$$
\kappa_{n}(h)=\frac{1}{2 \pi i} \int_{|z|=\rho} K\left(\frac{\gamma(z)}{h}\right) z^{-n-1} d z,
$$

and $\rho$ being the radius of a circle in the domain of analyticity of $K(s)$, (7) can be simplified by Cauchy's product of two series

$$
\sum_{n=0}^{\infty} \kappa_{n}(h) z^{n} \sum_{n=0}^{\infty} g(n h) z^{n}=\sum_{n=0}^{\infty} \sum_{j=0}^{n} \kappa_{n-j}(h) g(j h) z^{n} .
$$

Taking now the n-th coefficient of the power series (10), the quadrature formula reads

$$
f(n h) \approx f_{n}=\sum_{j=0}^{n} \kappa_{n-j}(h) g(j h) z^{n},
$$

for $n=0,1, \ldots, N$, where the integration weights $\kappa_{n}$ are determined by (9). By introducing the polar coordinate $z=\rho e^{i \theta}$, we have the following integral representation for these coefficients

$$
\kappa_{n}(h)=\frac{\rho^{-n}}{2 \pi} \int_{0}^{2 \pi} K\left(\frac{\gamma\left(\rho e^{i \theta}\right)}{h}\right) e^{-i n \theta} d \theta
$$

This integral can be efficiently computed by using the trapezoidal rule, that is,

$$
\kappa_{n}(h) \approx \frac{\rho^{-n}}{L} \sum_{l=0}^{L-1} K\left(\frac{\gamma\left(\rho e^{2 \pi i l} / L\right.}{h}\right) e^{-2 \pi i n / L},
$$

where the interval $(0,2 \pi)$ has been partitioned into $L$ subintervals of equal length. Once the weights $\kappa_{n}(h)$ and the required values of $g$ are computed, the discrete convolution (11) can be evaluated in $O(N \log N)$ arithmetical operations, using FFT .

Theorem 1. Let $f$ be defined as (2) where $K(s)$ is sectorial and the chosen multistep method is $A(\alpha)$ stable of order $p$, then

$$
\left|f_{n}-f\left(t_{n}\right)\right| \leq C t_{n}^{\mu-p-1} h^{p},
$$

where the constant $C$ does not depend on $h$ and $t_{n}$.
Proof. [1] To have the maximum convergence order $p$, Lubich in [1] has suggested to modify the quadrature rule as follows

$$
\tilde{f}_{n}=f_{n}+\sum_{j=0}^{p-2} c_{j} \kappa_{n-j}(h) g(j h),
$$

where $c_{j}, j=0, \ldots, p-2$, are the correction weights of the p-th order Newton- Gregory formula (end-point correction of the trapezoidal rule).

Theorem 2. Under the assumptions of Theorem 1, and for $g \in C^{p}[0, \infty)$, the method (14) satisfies

$$
\left|\tilde{f_{n}}-f\left(t_{n}\right)\right| \leq C^{\prime} h^{p},
$$

where the constant $C^{\prime}$ does not depend on $h$ and $t_{n}$.

\section{COllocation Method}

Consider the integral equations (1) for $i=1,2, \ldots, M$, let Laplace transform of $k_{i j}(x)$ are $K_{i j}(s)$, and assume that $K_{i j}(s), i, j=1,2, \ldots, M$, are sectorial.

Define $x_{n}=n h$ where $h=\frac{b}{N}$, by using the modified quadratare formula (14), we may write

$$
\begin{array}{r}
\int_{0}^{x_{n}} k_{i j}(x-t) \Phi_{i j}\left(t, f_{j}(t)\right) d t=\sum_{k=0}^{n} \kappa_{n-k}^{(i j)}(h) \Phi_{i j}\left(x_{k}, f_{j}\left(x_{k}\right)\right) \\
+\sum_{k=0}^{p-2} c_{k}^{(i j)} \kappa_{n-k}^{(i j)}(h) \Phi_{i j}\left(x_{k}, f_{j}\left(x_{k}\right)\right)+O\left(h^{p}\right),
\end{array}
$$

for $i, j=1,2, \ldots, M$, and $n=1,2, \ldots, N$, where

$$
K_{i j}\left(\frac{\gamma(z)}{h}\right)=\sum_{n=0}^{\infty} \kappa_{n}^{(i j)}(h) z^{n}
$$

At $x=x_{n}$, for $n=1, \ldots, N$, we have, on substituting (15) in $(1)$,

$$
\begin{aligned}
\sum_{j=1}^{M} q_{i j}\left(x_{n}\right) f_{j}\left(x_{n}\right) & =g_{i}\left(x_{n}\right)+\sum_{j=1}^{M}\left(\sum_{k=0}^{n} \kappa_{n-k}^{(i j)}(h) \Phi_{i j}\left(x_{k}, f_{j}\left(x_{k}\right)\right)\right. \\
& \left.+\sum_{k=0}^{p-2} c_{k}^{(i j)} \kappa_{n-k}^{(i j)}(h) \Phi_{i j}\left(x_{k}, f_{j}\left(x_{k}\right)\right)\right)+O\left(h^{p}\right) .
\end{aligned}
$$

So, the corresponding approximating equations are

$$
\begin{aligned}
\sum_{j=1}^{M} q_{i j}\left(x_{n}\right) f_{j n} & =g_{i}\left(x_{n}\right)+\sum_{j=1}^{M}\left(\sum_{k=0}^{n} \kappa_{n-k}^{(i j)}(h) \Phi_{i j}\left(x_{k}, f_{j k}\right)\right. \\
& \left.+\sum_{k=0}^{p-2} c_{k}^{(i j)} \kappa_{n-k}^{(i j)}(h) \Phi_{i j}\left(x_{k}, f_{j k}\right)\right),
\end{aligned}
$$

for $i=1,2, \ldots, M$, and $n=1,2, \ldots, N$.

Equations (16) determine a system of $N M$ nonlinear algebraic equations to be solved for $N M$ unknown coefficients $f_{j n}, j=1, \ldots, M, n=1, \ldots, N$.

\section{APPLiCATIONS IN PHYSics}

Parabolic partial differential equations with nonlinear 
boundary conditions appear in several branches of applied physics and engineering (cf. [6] and the references therein). For example, consider the heat conduction in a rod of length 1 along which heat may be dissipated. At one end of the rod energy is absorbed at a given rate and radiates nonlinearly according to Boltzmann' law while at the other end the energy radiates linearly or the temperature is kept at the same level as the surrounding temperature. Based on the balance relation of the heat conduction, the temperature $\mathrm{w}$ in the rod is governed by the one dimensional parabolic problem [6]

$$
\begin{gathered}
\frac{\partial w}{\partial t}-D \frac{\partial^{2} w}{\partial x^{2}}+C\left(w-\theta_{0}\right)=q(x, t), \\
w(x, 0)=w_{0}(x), \\
-w_{x}(0, t)+\sigma\left(w^{m}(0, t)-\theta_{0}^{m}\right)=h(t), \\
\alpha_{0} w_{x}(l, t)+\beta_{0}\left(w(l, t)-\theta_{0}\right)=0,
\end{gathered}
$$

where $0<x<l, 0<t<T, D, C$ and $\sigma$ are positive constants, $q, h$ and $w_{0}$ are smooth nonnegative functions representing the internal, boundary and initial source, respectively, and $\theta_{0} \geq 0$ denotes energy dissipation along the rod and $\sigma\left(w^{m}(0, t)-\theta_{0}^{m}\right)$ is due to the effect of nonlinear radiation.

Also, in many biochemical reaction processes such as fermentation, waste treatment, and production of pharmaceutical, the reaction rate of the concentration as often assumed to obey the Michaelis-Menton hypothesis. When this reaction appears at the boundary surface of the diffusion medium it leads to a nonlinear boundary condition. A simple model of the fermentation problem is given in the form (17)-(20) except with and with the $\theta_{0}=0$ boundary condition at $x=0$ replaced by

$$
-w_{x}(0, t)=\frac{\sigma w(0, t)}{1+a w(0, t)},
$$

where $\sigma$ and $a$ are positive constants.

Moreover, in the reaction diffusion process where gas is in contact with liquid the gas is absorbed by the liquid at the gad-liquid interface. If the diffusion medium is a slab and the interfacial absorption occurs at the slab face $x=0$ then the one-dimensional mathematical model for the liquid density $w$ is given by (17)-(20) with $C=\theta_{0}=0$ and with the boundary condition at $x=0$ replaced by

$$
-w_{x}(0, t)=\sigma\left(w^{-\delta}(0, t)-w(0, t)\right)
$$

where $\sigma$ and $\delta$ are positive constants.

Generally, consider the problem of determination of the function $w$ in the following parabolic problem

$$
\begin{gathered}
\frac{\partial w}{\partial t}=\frac{\partial^{2} w}{\partial x^{2}}+p_{1} \frac{\partial w}{\partial t}+p_{2} w+p_{3}(x, t), \\
w(x, 0)=w_{0}(x),
\end{gathered}
$$

$$
\begin{aligned}
& w_{x}(0, t)=f_{0}(t, w(0, t)), \\
& w_{x}(1, t)=f_{1}(t, w(1, t)),
\end{aligned}
$$

where $p_{1}$ and $p_{2}$ are constants and $p_{3}, w_{0}, f_{0}$ and $f_{1}$ are known functions.

Employing the transformation

$$
u(x, t)=w(x, t) \exp \left\{\frac{p_{1}}{2} x-\left(p_{2}-\frac{p_{1}^{2}}{4}\right) t\right\},
$$

the problem (23)-(26) will become [7]

$$
\begin{gathered}
\frac{\partial u}{\partial t}=\frac{\partial^{2} u}{\partial x^{2}}+q(x, t), \\
u(x, 0)=u_{0}(x), \\
u_{x}(0, t)=g_{0}(t, w(0, t)), \\
u_{x}(1, t)=g_{1}(t, w(1, t)) .
\end{gathered}
$$

Consider the problem (27)-(30) and assume that the source function $q(x, t)$ is bounded and uniformly Holder continuous on each compact subset of the domain. We shall also assume that the initial function $u_{0}$ is piecewise-continuous, $g_{0}$ and $g_{1}$ are Lipschitz- continuous with respect to $u$. So, the bounded unique solution $u$ of the problem is the form [7]

$$
\begin{aligned}
u(x, t)=v(x, t) & -2 \int_{0}^{t} \theta(x, t-\tau) g_{0}(\tau, \varphi(\tau)) \\
& +2 \int_{0}^{t} \theta(x-1, t-\tau) g_{1}(\tau, \psi(\tau)),
\end{aligned}
$$

where $\varphi$ and $\psi$ are piecewise-continuous solutions of the following system of integral equations

$$
\begin{aligned}
& \varphi(t)=v(0, t)-2 \int_{0}^{t} \theta(0, t-\tau) g_{0}(\tau, \varphi(\tau)) d \tau \\
& +2 \int_{0}^{t} \theta(1, t-\tau) g_{1}(\tau, \psi(\tau)) d \tau, \\
& \psi(t)=v(1, t)-2 \int_{0}^{t} \theta(1, t-\tau) g_{0}(\tau, \varphi(\tau)) d \tau \\
& +2 \int_{0}^{t} \theta(0, t-\tau) g_{1}(\tau, \psi(\tau)) d \tau, \\
& v(x, t)=\int_{0}^{1} G(x, t, \zeta, 0) u_{0}(\zeta) d \zeta \\
& +\int_{0}^{t} \int_{0}^{1} G(x, t, \zeta, \tau) q(\zeta, \tau) d \zeta d \tau, \\
& G(x, t, \xi, \tau)=\theta(x-\xi, t-\tau)+\theta(x+\xi, t-\tau),
\end{aligned}
$$

and

$$
\theta(x, t)=\frac{1}{2 \sqrt{\pi t}} \sum_{n=-\infty}^{\infty} \exp \left\{-\frac{(x+2 n)^{2}}{4 t}\right\} .
$$

Thus, the problem (27)-(30) is reduced to (31)-(33). So, by solving the system of nonlinear Volterra integral equations (32) and (33), we obtain $\varphi(t)$ and $\psi(t)$, then, if we replace them in (31), we will have the solution $u(x, t)$. 


\section{NuMERICAL RESUlts}

In this section, we illustrate the use of the proposed method by displaying the results obtained from its application to a test problem.

Example 1. Consider the system of integral equations (1) with

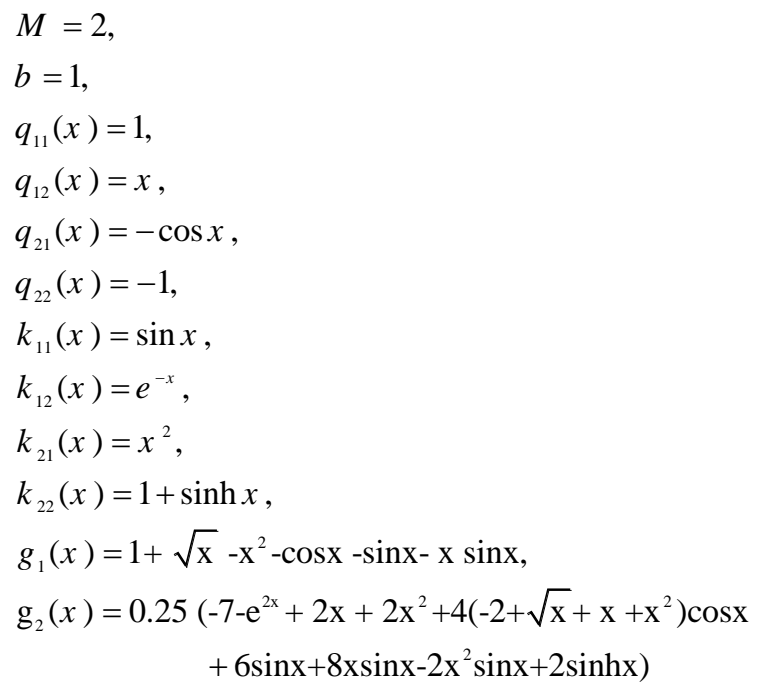

with the exact solution

$$
\begin{aligned}
& f_{1}(x)=\sqrt{x}, \\
& f_{2}(x)=-\sin x .
\end{aligned}
$$

The example has been solved by taking $p=2$ and different values of $N$. The exact and approximate solutions for $N=20$ and $N=50$ are shown in Fig. 1-Fig. 4.

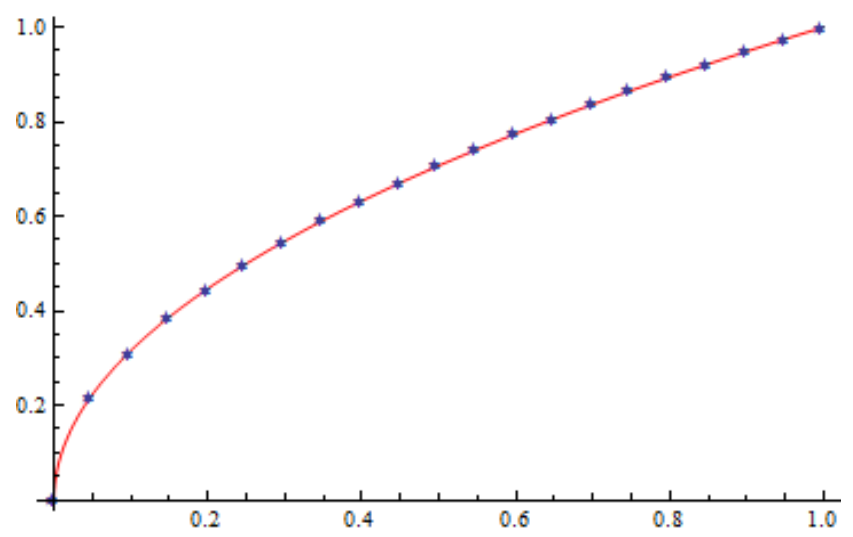

Fig. 1. Plot of exact and approximate solution of $f_{1}(x)$ for $N=20$.

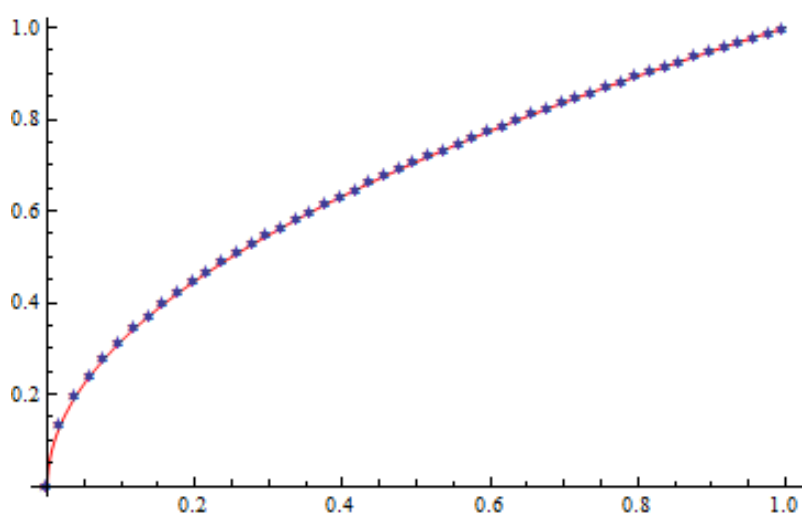

Fig. 2. Plot of exact and approximate solution of $f_{1}(x)$ for $N=50$.

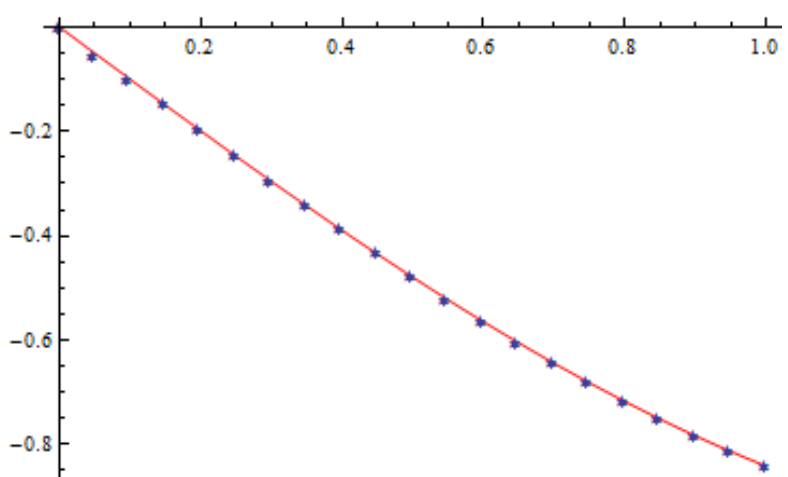

Fig. 3. Plot of exact and approximate solution of $f_{2}(x)$ for $N=20$.

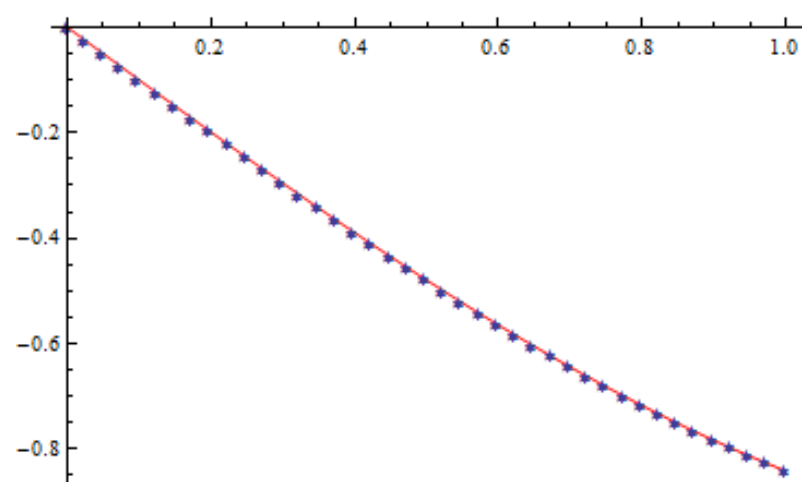

Fig. 4. Plot of exact and approximate solution of $f_{2}(x)$ for $N=50$.

TABLE I: ABSOLUTE ERRORS FROM THE TEST PROBLEM IN EXAMPLE 2

Exact

Error

\begin{tabular}{llll}
$(x, t)$ & $u(x, t)$ & $N=20$ & $N=40$ \\
\hline$(0.1,0.1)$ & -0.2905 & $3.2 \times 10^{-3}$ & $5.1 \times 10^{4}$ \\
\hline$(0.2,0.2)$ & -0.2063 & $3.4 \times 10^{-3}$ & $5.0 \times 10^{4}$ \\
\hline$(0.3,0.3)$ & -0.1300 & $2.9 \times 10^{-3}$ & $5.0 \times 10^{-4}$ \\
\hline$(0.4,0.4)$ & -0.0613 & $2.9 \times 10^{-3}$ & $4.9 \times 10^{-4}$ \\
\hline$(0.5,0.5)$ & 0 & $2.8 \times 10^{-3}$ & $4.8 \times 10^{-4}$ \\
\hline$(0.6,0.6)$ & 0.0542 & $2.7 \times 10^{-3}$ & $4.8 \times 10^{-4}$ \\
\hline$(0.7,0.7)$ & 0.1016 & $2.7 \times 10^{-3}$ & $4.7 \times 10^{-4}$ \\
\hline$(0.8,0.8)$ & 0.1425 & $2.5 \times 10^{-3}$ & $4.6 \times 10^{-4}$ \\
\hline$(0.9,0.9)$ & 0.1774 & $2.6 \times 10^{-3}$ & $4.4 \times 10^{-4}$ \\
\hline \hline
\end{tabular}

Example 2. Consider the following parabolic problem

$$
\begin{aligned}
& \frac{\partial u}{\partial t}=\frac{\partial^{2} u}{\partial x^{2}}, \\
& u(x, 0)=\sin \left(\frac{\pi}{4}\left(x-\frac{1}{2}\right)\right), \\
& u_{x}(0, t)-u^{4}(0, t)=h_{0}(t), \\
& u_{x}(1, t)-u(1, t)=h_{1}(t),
\end{aligned}
$$

which

$$
\begin{aligned}
& h_{0}(t)=\frac{1}{4} \exp \left\{-\frac{\pi^{2} t}{16}\right\} \pi \cos \frac{\pi}{8}-\exp \left\{-\frac{\pi^{2} t}{4}\right\} \sin ^{4} \frac{\pi}{8}, \\
& h_{1}(t)=\frac{1}{4} \exp \left\{-\frac{\pi^{2} t}{16}\right\} \pi \cos \frac{\pi}{8}-\exp \left\{-\frac{\pi^{2} t}{16}\right\} \sin \frac{\pi}{8},
\end{aligned}
$$

and is easily seen to have the exact solution 


$$
u(x, t)=\sin \left(\frac{\pi}{4}\left(x-\frac{1}{2}\right)\right) \exp \left\{-\frac{\pi^{2} t}{16}\right\}
$$

We report the absolute value of the errors of our method with $N=20,30,40$, at various points in Table I.

\section{REFERENCES}

[1] C. Lubich, "Convolution quadrature and discretized operational calculus. I," Numer. Math., vol. 52, pp. 129-145, 1988.

[2] C. Lubich, "Convolution quadrature and discretized operational calculus. II ," Numer. Math., vol. 52, pp. 413-425, 1988.

[3] C. Lubich, "Convolution quadrature revisited," BIT Numer. Math., vol. 44, pp. 503-514, 2004.

[4] C. Lubich, "Fractional linear multistep methods for Abel-Volterra integral equations of the second kind," Math. Comput., vol. 45, pp. $463-469,1985$.
[5] C. Lubich, "On the numerical solution of Volterra equations with unbounded nonlinearity," J. Integral Equations., vol. 10, pp. 175-183, 1985.

[6] C. V. Pao, Nonlinear parabolic and elliptic equations, New York: Plenum Press, 1992.

[7] R. Zolfaghari and A. Shidfar, "A numerical study of parabolic problems with nonlinear boundary conditions," Math Meth Appl Sci., vol. 35 , pp. 1312-1320, 2012.

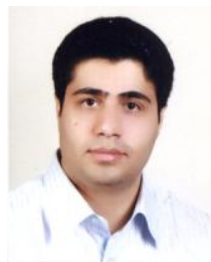

R. Zolfaghari was born in Nahavand, a small city of Hamedan satate in Iran, in February 1982. He received $\mathrm{BSc}$ in pure mathematics from Buali Sina University in 2005, MSc and PhD in applied mathematics from Iran University of Science and Technology in 2007 and 2011, respectively. During his graduation study, he worked on numerical methods for solving direct and inverse problems of Parabolic PDEs.

Now, he is the assistant professor in Department of Computer Science in Salman Farsi University of Kazerun, Iran. 